\title{
Optimizing Screening Colonoscopy: Strategies and Alternatives
}

\author{
Hans-Dieter Allescher Vincens Weingart \\ Department of Gastroenterology, Klinikum Garmisch-Partenkirchen, Garmisch-Partenkirchen, Germany
}

Keywords

Adenoma detection rate $\cdot$ Polyp detection rate $\cdot$ Interval carcinoma Colorectal cancer $\cdot$ Screening

\section{Abstract}

Screening colonoscopy is the most effective screening procedure for the prevention of colorectal cancer. The efficacy of colonoscopy is highly dependent on the overall quality of how this procedure is indicated, planned, prepared, and performed. The quality is directly linked to the number of polyps and/or adenomas detected or, in other words, to the number of polyps or adenomas missed during the procedure. The quality has a direct impact on the rate of interval carcinoma and on the range of how the incidence and occurrence of colorectal cancer is reduced. This review summarizes the current status on general measures and procedure improvements and standards as well as technical advances which have been suggested and established to improve the quality of polyp and adenoma detection rate. This includes selection and preparation of the patients, planning, methodological and technical performance of the procedure, and technical advances of the endoscope technology in order to improve screening results. It also covers new technologies with wide angle endoscopes (Ewave) and IT-based approaches using artificial intelligence to such as ai4Gl for the polyp detection and image analysis.

(c) 2019 S. Karger AG, Basel

\section{Introduction}

It has been suggested that the development of colorectal cancer is based on a graded stepwise mutation process [1] and that the majority of the carcinomas derive from adenomatous mucosal changes, which can be identified as flat or pedunculated polyps. This adenoma-carcinoma sequence has been the basic principle for the introduction of screening colonoscopy, where these mucosal abnormalities can be detected and removed before higher mutational states are reached. By removing these premalignant neoplastic polyps, the development of colorectal cancer can be prevented.

It is estimated that up to $80 \%$ of all colorectal cancers could be prevented by screening colonoscopy. It has been shown that screening procedures such as testing for occult blood (FOBT) or screening sigmoidoscopy will also decrease the expected cancer incidence. In 2002, a nationwide screening colonoscopy program was introduced in Germany and along with it a strict quality control program. Since the introduction of the screening program, more than 2 million colonoscopies have been performed and there is a small decrease in the overall incidence of colorectal cancer. It is estimated that in the period from 2003 to 2010, 100,000 cases of CRC have been avoided and 50,000 new cases have been diagnosed at an earlier stage [2]. The voluntary program is still limited by the overall participation, which so far has reached only 20$25 \%$ of all the eligible persons. Thus, additional programs such as an invitation program have been started to increase the acceptance of and participation in the screening program.

It has been shown in the past that the quality of the colonoscopy performed is crucial for the outcome of interval carcinomas and the overall prevention effect of this procedure. Therefore, multiple activities have been undertaken to optimize colonoscopy in its quality and in the polyp detection rate. Detection of polyps and especially of adenomas in the so-called adenoma detection rate

\section{KARGER}

(C) 2019 S. Karger AG, Basel
Prof. Dr. Hans-Dieter Allescher 
(ADR) is a major quality indicator for the effectiveness of this screening procedure [3].

More than 45,000 colonoscopies from 186 endoscopists were followed and the rate of interval carcinoma $(n=42)$ was determined over a period of 188,788 personyears. The individual ADR of the endoscopists was significantly associated with the risk of an interval carcinoma [3]. While the ADR showed an association for the occurrence of an interval carcinoma, the cecal intubation rate showed no correlation.

Evaluation of more than 300,000 colonoscopies performed by 136 gastroenterologists showed that every $1.0 \%$ increase in ADR was associated with a 3.0\% decrease in the risk of cancer (hazard ratio $0.97 ; 95 \%$ CI 0.96-0.98) [4]. Adenoma miss rates during screening colonoscopy are a serious problem and are estimated to be anywhere between $6-27 \%$. All efforts should be undertaken to reduce this rate. The present review will summarize various techniques to reduce the adenoma miss rates and increase polyp and ADR.

\section{Strategies to Improve Quality of Screening Colonoscopy}

\section{General Measures}

Patient Selection Based on Family History and

Genetic Risk

The first step in the general activities to improve the quality of screening colonoscopy is the adequate and optimal selection of eligible patients for colonoscopy. Patients with a higher risk and higher rate of polyp occurrence will benefit more from the screening as the likelihood of colorectal cancer is increased and there is an increased probability to detect polyps and adenomas.

At the moment, there are activities to identify genetic risk populations and familial risk factors for the development of colorectal cancer. Thus, a distinct family history applying the Amsterdam and modified Bethesda criteria is generally used. However, it is crucial to also perform genetic testing in sporadically occurring cancers especially in young patients, in patients with synchronous cancers, and in patients with characteristic histology. Besides the microsatellite instability and immune-histochemistry for mismatch enzymes, the newer genetic tests allow specified testing of these patients. This is not within the spectrum of the current review.

\section{Bowel Preparation}

The next important step is the overall preparation of the patient for the procedure and the cleaning of the large bowel for the colonoscopy procedure. Dietary recommendations are crucial for the preparation and avoidance of grains and special solids during the days before the procedure should be transferred to the patient.

There are new devices and apps using social media and messenger systems to support the patient in the bowel preparation. One of these apps gives useful advice and reminds the patient to drink the cleansing solutions [5] and was recently shown to improve bowel cleansing and $\mathrm{ADR}$ in the right colon [6]. The authors also report a high acceptance and lower discomfort of the bowel preparation. Additional information material helps to improve preparation quality especially in patients with foreign language. There are special patient groups (chronic constipation, spinal cord injury) which will need a more intense preparation program, but these patients have to be identified beforehand.

In general, various types and modifications of polyethylene glycol- or macrogol-containing solutions with or without electrolytes and or taste modifiers (lemon, ascorbic acid) are used for cleaning the bowel and the mucosal surface. It has been shown that suboptimal preparation of the bowel decreases the yield of polyp and adenoma detection, and up to $48 \%$ of the adenomas present will be missed, including also advanced adenoma with a size larger than $10 \mathrm{~mm}$.

More importantly, most of the missed adenomas are located in the proximal right-sided colon (64.8\%). This could explain the fact that colonoscopy is less effective in the right-sided colon, and in some studies, it was not possible to show a reduction of the incidence of CRC in the proximal right-sided colon. In addition, bowel cleaning is the single independent negative factor for reduction of the cecum intubation rate [7].

In the Modec study, the impact of bowel cleansing on the efficacy of colonic cancer screening was valuated in a prospective randomized study. Proper cleaning of the colonic surface increased the detection rate of adenomatous polyps in the right side and transverse colon [8].

In fact, in most guidelines including the German Gastroenterological Association S2K guideline, it is emphasized that a proper screening colonoscopy can only be performed if the bowel preparation is sufficient. Therefore, it is mandatory to document the degree of bowel contamination, usually with the Boston Bowel Preparation Scale (BBPS) or the Harefield Cleansing Scale.

In addition to the oral drinking preparation, there are additional devices which can be used to remove remnants from the mucosal surface such as a water jets through the working channel or through an additional jet channel of the endoscope to optimize bowel preparation.

The goal should be an inspection and evaluation of more than $95 \%$ of the mucosal colonic surface. There are some virtual reality programs which render the mucosal surface and control and estimate the amount of the mucosal surface inspected and evaluated. 
Patient's Position: Change of the Patient's Position/

Posture during Endoscopy

There is an ongoing debate whether colonoscopy should be performed in a supine or in a left-sided position. Usually, this is done at the endoscopist's preference. In most cases, the endoscope is introduced in a left-sided position and advanced to the sigma or descending colon. Then, some endoscopists turn the patient into a supine position, whereas others use the stable left-sided position to advance the endoscope as far as possible.

An argument for the use of the supine position is the fact that the left-sided descending colon will not be compressed by the abdominal content and thus advancement and inspection is facilitated. The disadvantages of the supine position are increased respiratory movements and increased rate of choking and respiratory problems. In the left-sided position, the inspection of the right-sided colon and the intubation of the terminal ileum seems facilitated.

The aspect of the colon surface is irregular due to muscular folds, which are generated by slowly propagating muscular contractions. As there are blind regions behind the folds, it is recommended that a given segment should be re-inspected repetitively to omit the blind areas behind the folds in order to see as much mucosal surface as possible. There are different technical alternatives (EndoCuff, G-eye, wide angle endoscopy, third eye endoscopy) which also aim to overcome these blind spots.

In order to improve the inspection of the mucosal surface, some authors further suggest a programmed repositioning of the patient (cecum, ascending colon: left-sided, transverse colon: supine, descending and sigmoid colon and rectum: right-sided position) increased the ADR per patient from 33 to $42.4 \%$ and the number of adenomas per patient increased from 0.67 to 0.9 [9]. This effect was most pronounced in the transverse and left-sided colon. However, this effect could only be observed in less experienced colonoscopists with a lower ADR, whereas in experienced endoscopists with a high ADR, programmed repositioning of the patient did not further increase the ADR [9].

Another method to reduce the muscular mucosal folds is the use of a spasmolytic such as butylscopolamine to flatten the colonic surface; however, despite the fact that this is commonly used, it did not increase the ADR during routine colonoscopy. Despite the fact that butylscopolamine or other antimuscarinergic spasmolytics flatten the folds and facilitate the mucosal inspection and the intubation process, they were also associated with side effect such as tachycardia $[10,11]$.

Time for Withdrawal

The quality of colonoscopy increases with the percentage of the mucosal surface inspected. Therefore, the time used to inspect the mucosal surface is crucial for the ADR.

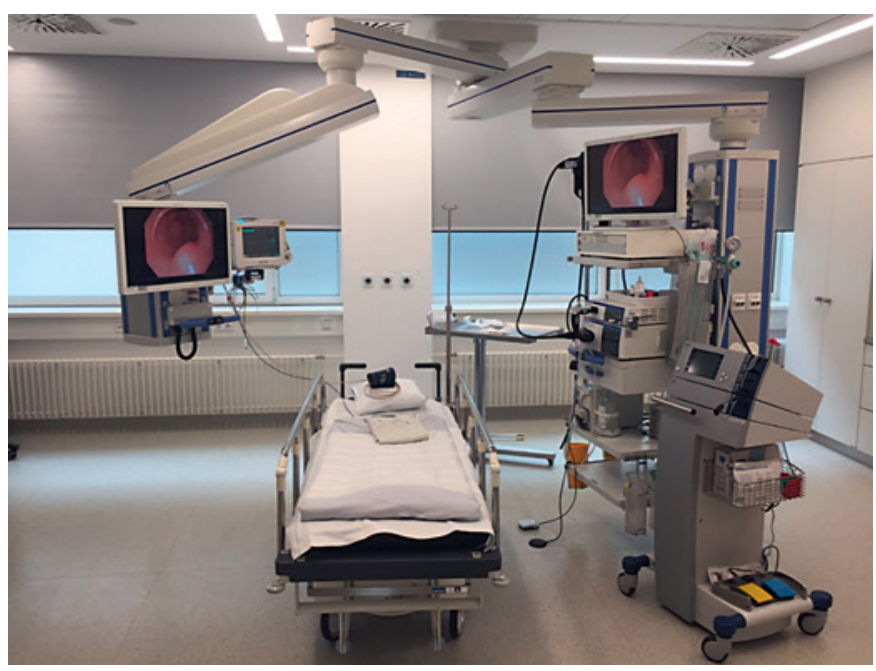

Fig. 1. Design of the endoscopic suite with two monitors to assist the endoscopy nurse personnel acting as second observer during colonoscopy.

If the withdrawal time of the endoscope was lower than 6 min, the ADR was below $11.8 \%$. However, if the withdrawal time was more than $6 \mathrm{~min}$, the ADR was more than double $(28.3 \%, p<0.001)$ [12]. Based on this key point study by Barclay et al. [12], the recommendation in the German endoscopy quality guideline is that the pure withdrawal time, without time used for biopsies or polypectomies, should be documented and should be longer than 6 min [13]. When the effect of withdrawal time was analyzed in a large database collective of more than 75,000 colonoscopies, it could be shown that each additional minute of withdrawal time adds an increase in the ADR of $3.6 \%$. In addition, the medium withdrawal time is inversely linked to the incidence of interval carcinomas [14].

\section{Endoscopy Nurse Personnel as Second Observer} during Colonoscopy

As the evaluation of the colonic mucosa needs continuous optical attention to analyze the video image, it seemed plausible that some lesions might be missed by the endoscopists. To overcome this, the additional effect of using the endoscopy nurse personnel as a second observer during colonoscopy was studied and showed that this four-eye approach will further increase the ADR (Fig. 1). In a study with 844 colonoscopies, additional observation by the nurse personnel increased the polyp detection rate by $58 \%$ and the ADR by $47 \%$. However, this effect was achieved with experienced endoscopy nurses (>2 years of experience) and was only observed in less trained fellows and unexperienced endoscopists with less than 500 colonoscopies performed. It showed no additional effect in experienced endoscopists with a preexisting high ADR [15]. 
This finding was analyzed in several subsequent studies where this effect was either less pronounced or could not be demonstrated. In a large study, additional observation by the endoscopy nurse personnel increased the polyp detection rate by only 1.29 [16].

However, these results could be interpreted to suggest that the ability to continuously monitor a large video screen with a fast-moving live video is limited and so other methods to support the endoscopist could be helpful. These findings also have an impact on recent technologies where the viewing field is either increased or even split on several separated monitors. Despite the fact that this increases the overall surface inspected, it might be limited by the ability to evaluate several monitors with different viewing angles and thus could require an additional observer.

Vigilance of the Endoscopists

The previous studies have already emphasized the importance of the vigilance and attention of the endoscopist for observing the video images. In this context, it is interesting that the ADR achieved is depending on the working hours and duration of uninterrupted endoscopies. Sanaka et al. [17] compared the ADR in 3,619 screening colonoscopies with respect to working and day time. In the period from 8:00 to 12:00 o'clock, the ADR was $29.3 \%$, whereas in the afternoon from 12:00 to 18:00 o'clock, the detection rate was significantly lower $(25.3 \%, p<0.001)$. In fact, there was a trend for a reduction of the ADR with every hour of uninterrupted endoscopy. This indicates that the attention of the endoscopist is a very important parameter for the detection of polyps during colonoscopy. Endoscopists working in 4-hour shifts showed no difference between morning or afternoon hours.

\section{Endoscopy Technique}

In the past, there have been several studies which investigated the endoscopic miss rates of adenomas and small polyps during screening colonoscopy in a back-toback approach. Depending on the population studied and the protocol applied, the adenoma miss rate in routine colonoscopy varies from 15 to $35 \%$ missed adenomas. Several attempts have been made to lower this adenoma miss rate.

One method to increase the ADR and lower the adenoma miss rate to is improve the endoscopic technique. This involves the endoscope itself, the video system of the endoscope image moving from conventional to high definition video systems, and the use of optical improvements such as NBI, I-Scan, or FICE or the use of additional devices attached to the endoscopic tip. All these methods either increase the percentage of mucosal surface, which can be inspected with the endoscope, or the recognition and detection of the polypoid structures.

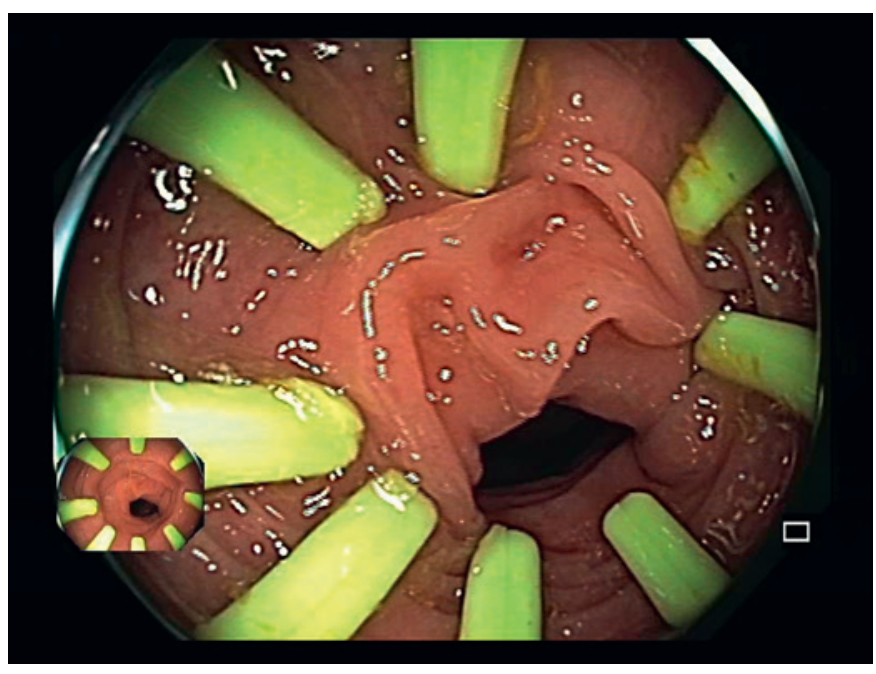

Fig. 2. Endoscopic image of colonoscopic appearance of the mucosal fold using an EndoCuff device. The green flaps retract and stretch the mucosal folds.

\section{Cap-Assisted Endoscopy}

As a first step, there was the suggestion to attach a transparent hood or cap to the tip of the endoscope. This device improves and stabilizes the constant distance of the endoscopic tip to the inspected mucosal surface. Use of a cap-assisted endoscopy increased the polyp detection rate from $39.1 \%$ without cap to $44.7 \%$ with a 2 -mm cap and $49.3 \%$ with a 4 -mm transparent hood cap. The authors concluded that the attachment of a transparent cap to the tip of the endoscope would increase the polyp detection rate [18]. This very promising initial result could only partially be reproduced and a subsequent large meta-analysis of all randomized studies with cap-assisted endoscopy showed only a small marginal increase in the polyp detection rate of $1.08[19,20]$. Thus, under some circumstances, the use of a cap can be helpful for the detailed inspection of a defined region.

\section{EndoCuff}

However, the results and experience of cap-assisted endoscopy led to the development of flexible devices attached to the endoscopic tip, which will retract the mucosal colonic folds. The first device, the EndoCuff, used small rubber flaps incorporated in a plastic cap fixed to the endoscopic tip. These flaps do not impose any resistance when advancing the endoscope, but they fold and bend retrogradely when the endoscope is retracted. With the circular orientation of the flaps, they flatten and stretch the mucosa, so that the mucosal folds are pulled into a flat position (Fig. 2). This allows a more complete inspection of the mucosa.

An initial study analyzed 200 patients in a back-toback colonoscopy study design with and without EndoCuff attached. The adenoma miss rate without EndoCuff 
was $38.4 \%$ in the whole colon and showed a high rate (38.9\%) especially in the right colon. With the EndoCuffassisted colonoscopy, the overall adenoma miss rate was only $14.7 \%$ and the adenoma miss rate in the right colon decreased to $10.4 \%$. Thus, the EndoCuff significantly increased the ADR and simultaneously decreased the adenoma miss rate in a back-to-back colonoscopic study approach [21]. In a similar study, the EndoCuff increased the ADR from 48.7 to $65 \%$ by $17 \%$ [22].

When analyzing the adenoma miss rates, EndoCuff reduced the adenoma miss rates by $63 \%$ and the polyp detection miss rates by $86 \%$ and increased the polyp detection rate [22-24]. Thus, the use of EndoCuff-assisted endoscopy is recommended for optimal screening; however, prospective studies showing the benefit still need to be done.

\section{Endorings}

Endorings ${ }^{\circledR}$ (Endopic EndoAid Ltd., Caesarea, Israel) are based on a similar principle and consist of overlapping transparent circular rings attached to the tip of the endoscopes. When the endoscope is pulled back, the rings stretch the mucosa and flatten the colonic folds. With this device, it is also possible to increase the visible surface of the colonic mucosa (Fig. 3). In a multicenter randomized tandem back-to-back colonoscopy study, Endorings decreased the adenoma miss rate from 48.3 to $10.4 \%$ and lowered the polyp miss rate from 52.8 to $9.1 \%$. Thus, also the results of the Endoring device support the results obtained with the EndoCuff principle: the stretching and flattening the mucosal folds will significantly reduce the adenoma miss rate and improve the detection rate [25].

\section{G-Eye Endoscopy}

The G-Eye technology uses another principle to stabilize and flatten the colonic mucosal folds. A flexible rubber balloon is constantly attached and incorporated to the tip of the endoscope, which can be inflated when the endoscope has reached the cecum (Fig. 4). Then, the endoscope is slowly retracted with the balloon inflated. The balloon stretches the mucosal folds and the visualization of the mucosal surface is improved. Using the G-Eye principle, a polyp detection rate of $53.2 \%$ and ADR of $44.7 \%$ could be achieved. However, no comparison to a standard endoscope in a back-to back study protocol was reported.

In general, the G-Eye technique additionally offers a more stable position of the endoscope [26-28].

\section{Improving Video Image}

With the introduction of video endoscopy, the quality of endoscopic image improved dramatically. The improvement in chip technology and the advancement of normal video image to high-definition white light (HD- 

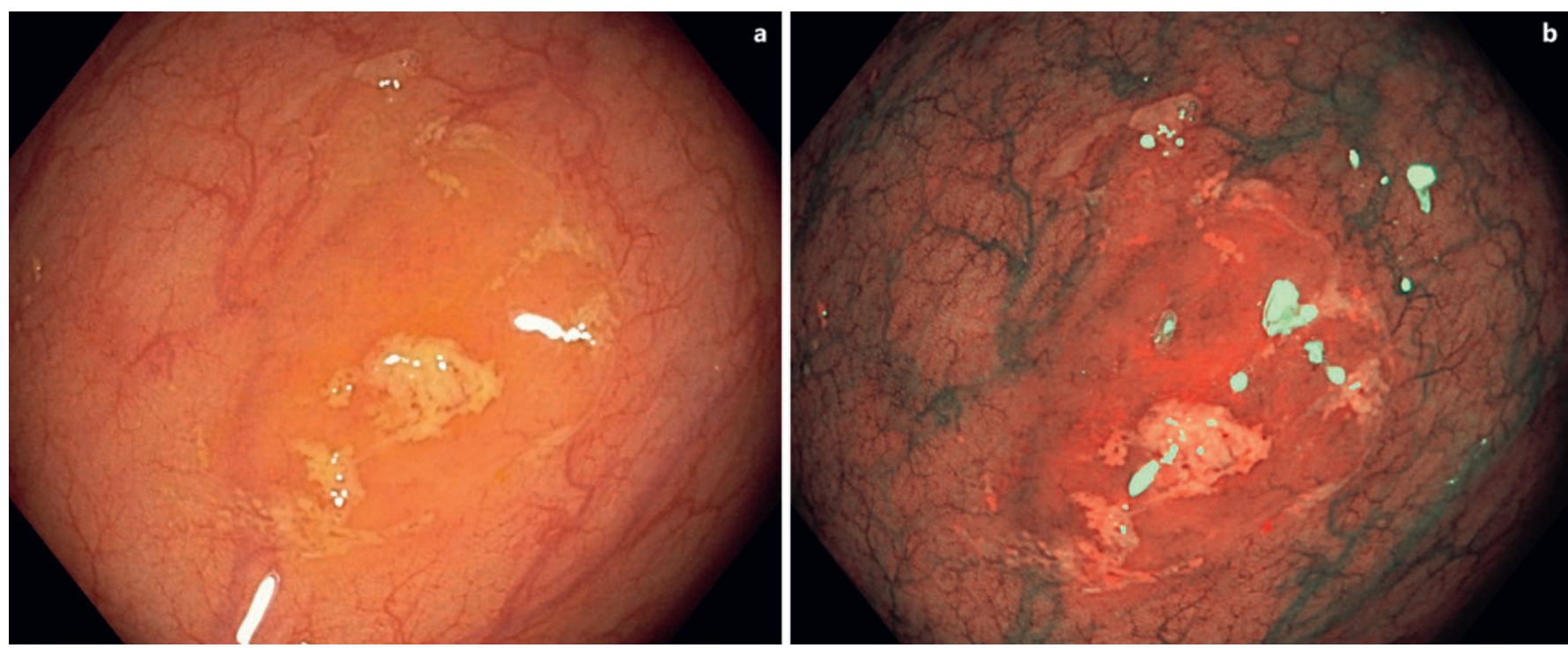

Fig. 5. a Endoscopic image of a serrated adenoma of the ascending colon with the typical white light appearance. The endoscopic image shows a slightly elevated flat lesion with a typical yellowish color and a mucous cap on top. $\mathbf{b}$ Endoscopic image of this typical serrated adenoma using NBI-II showing a typical reddening of the adenoma as an atypical vascular pattern.

tal cancers. In contrast, virtual chromoendoscopy using image-enhancing technology (FICE, I-SCAN) or selected bandwidth of the illuminating light (NBI, NBI-II) has no significant effect on the adenoma or polyp detection rates [30].

Improvement in overall video quality such as $\mathrm{HD}$-video endoscopy compared to common white light endoscopy increases the ADR. Additionally, when compared to conventional video endoscopy of a former technology, HD-WL endoscopy increases the diagnostic yield.

With HD-WL endoscopy, the additional use of NBI-II increased the ADR by $14 \%$ (Fig. 5) [31].

\section{Third Eye}

As already mentioned, normal prograde endoscopy might miss some polyps which are hidden behind mucosal folds and bends. In order to overcome this problem, several solutions have been suggested to visualize these blind spots for diagnostic evaluation. Besides the mechanical options such as caps, cuffs, balloons, and rings, there are also imaging technologies which could solve this problem.

The third eye technology uses a second endoscopy which is advanced through the working channel and is used in a retroflexed position to view the mucosal areas behind the folds [32]. The use of the third eye technology was shown to increase the $\mathrm{ADR}$ and polyp detection rate and to reduce the percentage of missed polyps. Even though this technology showed its benefits, it blocks the working channel and is technically demanding. It also forces the endoscopist to evaluate a second monitor,

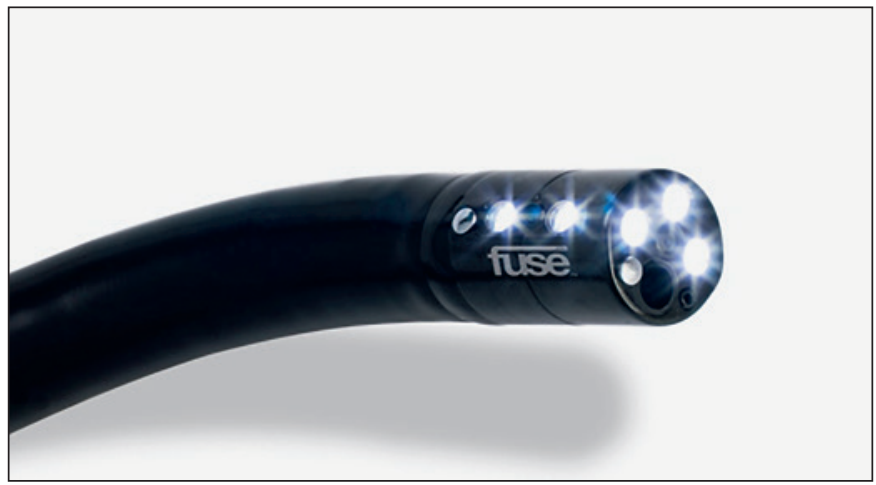

Fig. 6. Endoscopic tip of the full-spectrum endoscope with the LED light sources and the individual video chips in the prograde and side viewing direction.

which will limit his attention on the primary monitor. So far, this technology has not gained broad acceptance in the routine screening colonoscopy.

\section{FUSE - Full-Spectrum Endoscopy}

As the viewing angle and the inspection area is of crucial importance for the ADR, a new concept for future colonoscopes has been suggested, which involves the use of multiple video chips built into the tip of an endoscope allowing an almost 360-degree view. The individual images were displayed on 3 separate video monitors. The FUSE endoscopic technology also included other advances of endoscopic technology such as LED lighting (Fig. 6). In several studies, the advantages of the increased viewing range have been shown [33]. The major disadvantage was 

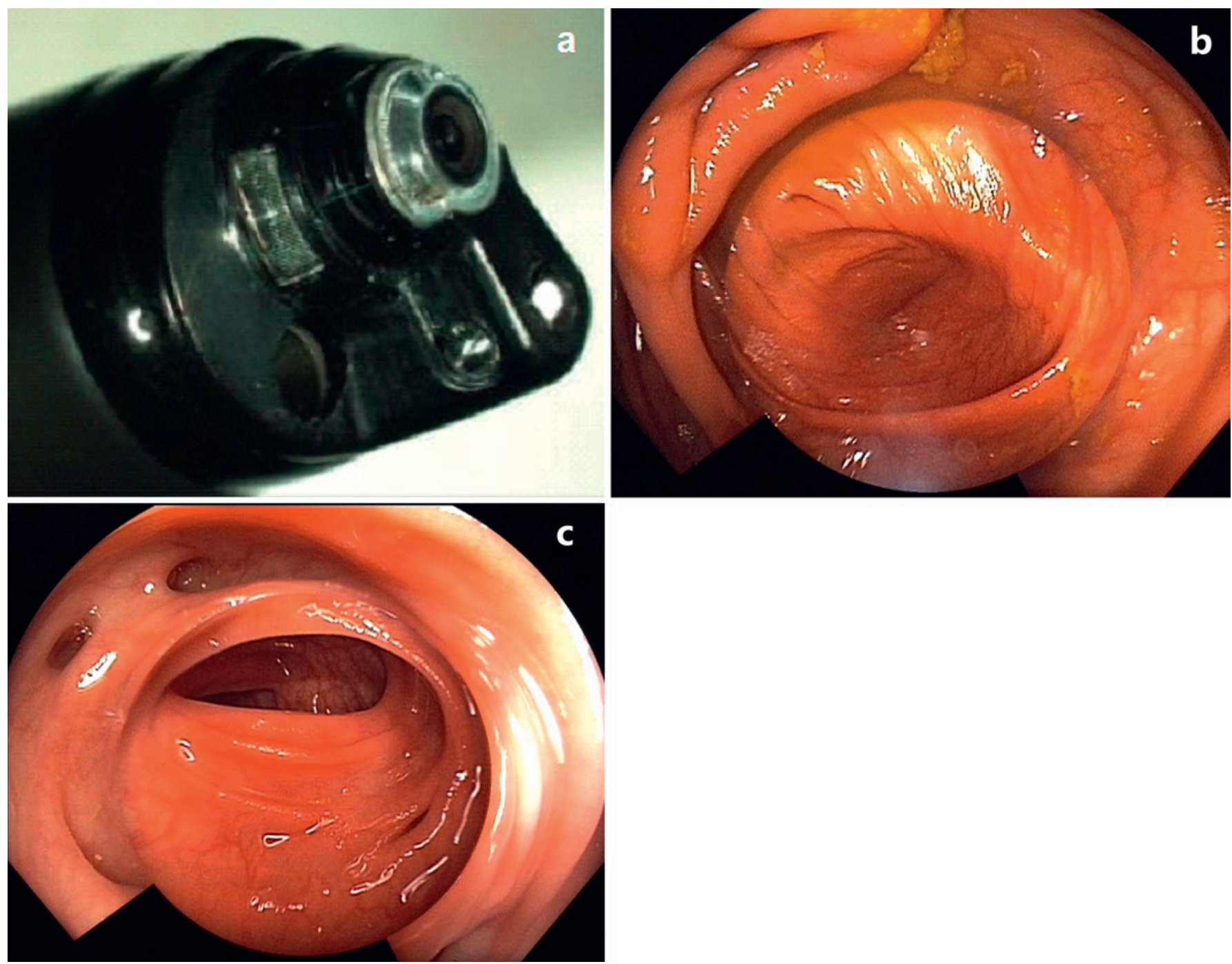

Fig. 7. a Tip of the EWAVE endoscope with the prograde view and the two backward oriented lenses. b, c Image examples of the EWAVE endoscope with the central forward view aspect and the outer side and backward viewing image.

the simultaneous evaluation of three video images on separate monitors. Unfortunately, this interesting new and technically advanced endoscopic system is currently no longer available.

EWAVE (Extra Wide-Angle View)

With this technology developed by Olympus, the endoscope offers a $235^{\circ}$ view obtained from a regular forward-viewing image and two lateral backward-viewing lenses, which are incorporated in the endoscopic tip. Both images are fused into a single endoscopy image, which allows a prograde view and on the side a backward view similar to a rear view mirror in cars (Fig. 7). The feasibility, safety, and clinical benefit of this technology was shown in a prospective multicenter cohort study in 193 patients with increased risk of colorectal polyps and/or cancer by experienced and EWAVE-trained experienced endoscopists. The EWAVE colonoscopy had a polyp detection rate of $61.1 \%$ (118/193), ADR of 39.9\% (77/193), and advanced ADR of 13.5\% (26/193) [19]. A randomized controlled study is missing to show the additional benefit of the EWAVE technology compared to standard HD-WL endoscopy.

\section{Automated Polyp Detection and Artificial} Intelligence

In the last years, there has been increasing interest to support the endoscopist's analysis of the live video image in order to improve the polyp detection by an analysis routine which would allow automatic polyp detection. This automated detection is influenced by several parameters such as endoscope/camera motion, light reflections 
from the mucosa, lack of focus, the commonly used wideangle lens, and the extreme variations in polyp size and positioning and morphology of the polyp. In addition, bubbles, fecal material, and other remnants as well as mucosal abnormalities may serve as false positive polyps.

Whereas older approaches used algorithms for polyp detection and structure analysis, the newer approaches are based on the principles of deep learning and pattern recognition from big data analysis. Using this approach, real-time optical detection and analysis of polyps is possible with white-light endoscopy alone, but can also be combined with advanced, endoscopic imaging modalities such as chromoendoscopy, NBI, endocytoscopy, or laserinduced autofluorescence spectroscopy [34].

\section{Machine Learning with Explicit Features}

The initial software tools for polyp detection (e.g., CoLD - Colorectal Lesions Detector) used second-order statistics and calculated the wavelet transformation of each image to discriminate normal from abnormal tissue. Using the abilities of an artificial neural network, the classification of these features, obtained from still images, achieved a detection accuracy of more than $95 \%$.

In a second approach, Fernández-Esparrach et al. [35] used energy maps based on localization of polyps and their boundaries (Window Median Depth of Valleys Accumulation [WM-DOVA] energy map method). This method reached a sensitivity of $70.4 \%$ and a specificity of $72.4 \%$ in video images for detection of polyps. As another approach, Wang and colleagues [36] used a rule-based classification and edge visual features to detect "polyp edges" (Polyp-Alert software). This system achieved a correct polyp detection in $97.7 \%$ with very little latency but had a considerable false positive rate [36].

Thus, in Japan, the first automated system for polyp detection and characterization (EndoBrain) has been approved in 2018 for the automated detection of polyps [37]. This system uses artificial intelligence or machine or deep learning to analyze the polyp structure, the mucosal surface, and the vascular pattern in order to predict the polyp histology and give advice for the treatment. In fact, this system reaches a negative predictive value of $>95 \%$ [37].

Another system uses image analysis (ai4GI) to give the probability of a polyp occurrence on the present endoscopic image and then uses the conventional and NBI-II image to classify the polyp into NICE-I and NICE-II histology [38]. These computerized systems are just emerging and the basic principle needs further images and comparative data, which will further increase the sensitivity and accuracy of these systems. Thus, a major advancement of these image analysis capabilities has to be expected in the near future, which will support the endoscopists.
A different approach is used by the automated polyp detection software (APDS), which uses a data reduction and weighted combination of color, structure, texture, and motion to detect image areas with possible polyps [39]. The APDS software reached an overall polyp detection rate of $75 \%$ but missed several adenomatous polyps and still had a considerable number of false positive hits. Despite these limitations, all these systems indicate that they are starting to assist the endoscopists in the current image analysis. As the "big data" approaches will improve with the number of polyps analyzed and the amount of data available, these systems will further improve and will help and eventually take over the image analysis of the endoscopic image leading to an automated colonoscopy.

\section{Computer-Assisted Diagnosis und Artificial}

Intelligence

In contrast to the automated polyp detection, there is the computer-assisted diagnosis (CAD) where the optical features of a detected lesion such as the pit pattern or vascular pattern would then allow the polyp classification such as the Kudo pit-pattern or the NICE classification. The Kudo pit-pattern is one of the most widely known classification systems used to classify and predict the histopathology of a given lesion [40].

Mori et al. [41] developed a machine-learning CAD system (EC-CAD system) that uses nuclear segmentation and feature extraction to predict pathologic classification. In a pilot study in still images, the system had a sensitivity of $92.0 \%$ and a specificity of $79.5 \%$. The sensitivity was within the range of expert endoscopist (92.7\%), whereas endoscopists reached higher specificity (91\%) [41]. In further advancement, Misawa developed a machine learning CAD system (AI-assisted endocytoscopy) to analyze endocytoscopy-NBI images [37, 42].

\section{Alternative Ways for Colorectal Cancer Screening Stool-Based Tests}

There are different test methods for testing individual stool specimens for the presence of occult blood as a screening for colorectal polyps, premalignant lesions, and carcinomas. The most common and usual stool-based method is the detection of occult blood (FOBT - fecal occult blood test) by guaiac paper. The sensitivity of this test method is very low, between $20-40 \%$. Furthermore, the test is affected by different circumstances: false positive results can be aroused by consumption of lots of red meat or vegetables with peroxidase activity, e.g., cauliflower, tomatoes, or radish, or by micro-bleeding in the upper GI tract caused by NSAR. False negative results can be caused by oral application or consumption of vitamin C (ascorbic acid).

Better sensitivity in detecting blood in the stool is possible with the hematoporphyrin test. This test method 
does not interfere with peroxidase but is still affected by bleeding in the upper GI tract.

The immunochemical tests show an obviously better sensitivity of $60-90 \%$. This test is detecting stable parts of hemoglobin or even special enzymes, for example pyruvate kinase. Certainly, these test methods are more expensive; however, this disadvantage is counterbalanced by a higher sensitivity.

Colonoscopy is the gold standard in diagnosing colorectal cancer. Nevertheless, stool tests are an alternative in screening for colorectal cancer, when colonoscopy is definitely refused. It should be implemented every year from 50 years without familial genetic strain (e.g., polyps or cancer in first-degree relatives).

\section{Virtual Colonoscopy}

CT-based colonography is an alternative diagnostic tool to examine the colon and rectum. Pre-diagnostic preparation of the colon is necessary compared to endoscopic colonoscopy. Solid components have to be washed out of the colon as much as possible to reduce artifacts. During examination, a distension of the colon is necessary by room air or carbon dioxide. Preparation is very similar to traditional colonoscopy. The examination duration of common CT-based colonography is about 10$15 \mathrm{~min}$. Intravenous contrast is not necessary but can offer, in combination with low-dose spiral abdominal CT exams, even more information beside colorectal disorder. The high-resolution scans are reconstructed by modern software to $2 \mathrm{D}$ and $3 \mathrm{D}$ pictures. Switching between the $3 \mathrm{D}$ endoscopic view and the corresponding $2 \mathrm{D}$ images for the reading physician is possible by flexible screen layouts and dual monitor system.

Sensitivity for detection of adenomas in a regular screening setting is depending on their size. It increases from $43 \%$ for small and diminutive polyps (polyps $<5$ $\mathrm{mm}$ ) to $61 \%$ for polyps ranging from 6 to $9 \mathrm{~mm}$ and $81 \%$ from polyps of $10 \mathrm{~mm}$ and larger [43].

\section{Patient Selection}

Finally, the most efficient approach to improve screening quality will be the selection of suitable patients and patients at risk. This involves characterization of risk scores and the use of blood and eventually stool or urine tests for the selection of patients at risk.

There are two genetic screening tests at the moment which will detect free DNA in the blood stream of the patient with analyses for single nucleotide polymorphisms, which put the patient at risk to develop polyps or to predict which polyp is at risk to further advance to colorectal cancer. The approach to detect and evaluate cell-free DNA (cfDNA) will fundamentally change our approach to screening from gastrointestinal tumor and will identify risk group patients and patients at high risk for the development of polyps and colorectal cancer. Highly sensitive and specific methods have been developed to detect ctDNA (BEAMing, Safe-SeqS, TamSeq) and to detect single nucleotide mutations in ctDNA.

In principle, the technologies can be divided into targeted approaches aimed to detect mutations in a set of predefined genes (e.g., KRAS proto-oncogene, GTPase [KRAS] in the context of EGFR [epidermal growth factor receptor] blockade by antibodies) or untargeted approaches (e.g., array-CGH [comparative genomic hybridization]). These approaches will be discussed in more detail in the following chapters [44-47].

\section{Conclusion}

Screening colonoscopy is currently the most powerful tool for the prevention of colorectal cancer. Together with the screening for occult blood, this screening procedure lowers the incidence and mortality related to colorectal cancer.

The screening effect is highly linked to the quality of how the colonoscopy is performed and to the resulting rate of adenomatous polyps and the percentage of missed polyps. In order to establish a high quality and ADR, the bowel preparation should be optimal and a Boston bowel preparation scale of 7-9 should be reached in $>90 \%$ of the patients. The technical experience of the endoscopist should allow a cecal intubation rate of $>95 \%$ and the technical success should be documented by photo documentation of the appendix orifice and the ileo-cecal valve. In a normal screening population, an ADR in men $>30 \%(35 \%)$ and an ADR in women $>20 \%$ (25\%) should be reached. The procedure time should be documented and the net withdrawal time excluding the time for biopsies and polyp removal should be $>6 \mathrm{~min}$. There should be a limitation of working hours of continuous coloscopy as the ADR decreases and all possibilities to improve the ADR (latest endoscope technology, integration of the endoscopic nurse, use of EndoCuff) and eventually in the future the use of an automated image analysis should be used. All optical improvements tend to increase the ADR; however, the major limitation is the visualization of blind spots behind folds and colonic curves. Here, wide-angle video devices and multiple video images could solve the problem. However, this is probably limited by the amount of video information which can be evaluated by a single endoscopist. Therefore, automated devices which will help to analyze video images and generate automated polyp detection or with alarm function could solve the problem. These devices can further be used for computer-assisted diagnosis which will help to predict or ever determine the histology of a given polyp.

The endoscopic methods are in continuous competition with other screening modalities. In the future, high- 
ly sensitive genetic methods could substitute for invasive screening procedures, thus decreasing the percentage of patients with normal finding or no detected polyps and limiting the patients to those with a high probability for a polyp. Thus, the screening colonoscopy could change to a preventing therapeutic procedure.

\section{Disclosure Statement}

The authors have no conflicts of interest to declare.

\section{References}

1 Vogelstein B, Fearon ER, Hamilton SR, Kern SE, Preisinger AC, Leppert M, et al. Genetic alterations during colorectal-tumor development. N Engl J Med. 1988 Sep;319(9): 525-32.

2 Brenner H, Hoffmeister M, Arndt V, Stegmaier C, Altenhofen L, Haug U. Protection from right- and left-sided colorectal neoplasms after colonoscopy: population-based study. J Natl Cancer Inst. 2010 Jan;102(2):89-95.

3 Kaminski MF, Regula J, Kraszewska E, Polkowski M, Wojciechowska U, Didkowska $\mathrm{J}$, et al. Quality indicators for colonoscopy and the risk of interval cancer. N Engl J Med. 2010 May;362(19):1795-803.

4 Corley DA, Levin TR, Doubeni CA. Adenoma detection rate and risk of colorectal cancer and death. N Engl J Med. 2014 Jun;370(26): 2541.

5 Walter BM, Klare P, Neu B, Schmid RM, von Delius S. Development and Testing of an $\mathrm{Au}$ tomated 4-Day Text Messaging Guidance as an Aid for Improving Colonoscopy Preparation. JMIR Mhealth Uhealth. 2016 Jun; 4(2):e75.

6 Walter B, Klare P, Strehle K, Aschenbeck J, Ludwig L, Dikopoulos N, Mayr M, Neu B, Hann A, Mayer B, Meining A, von Delius S: Improving the quality and acceptance of colonoscopy preparation by reinforced patient education with short message service: results from a randomized, multicenter study (PERICLES-II). Gastrointest Endosc. 2019 Mar; 89(3):506-513.e4.

7 Hsu CM, Lin WP, Su MY, Chiu CT, Ho YP, Chen PC. Factors that influence cecal intubation rate during colonoscopy in deeply sedated patients. J Gastroenterol Hepatol. 2012 Jan; 27(1):76-80.

8 Pohl J, Halphen M, Kloess HR, Fischbach W. Impact of the quality of bowel cleansing on the efficacy of colonic cancer screening: a prospective, randomized, blinded study. PLoS One. 2015 May; 10(5):e0126067.

9 Lee SW, Chang JH, Ji JS, Maeong IH, Cheung DY, Kim JS, et al. Effect of Dynamic Position Changes on Adenoma Detection During Colonoscope Withdrawal: A Randomized Controlled Multicenter Trial. Am J Gastroenterol. 2016 Jan;111(1):63-9.

10 Ristikankare M, Karinen-Mantila H. The role of routinely given hyoscine-N-butylbromide in colonoscopy: a double-blind, randomized, placebo-controlled, clinical trial. Scand J Gastroenterol. 2016 Mar;51(3): 368-73.
11 Jung P, Park SB, Kim HW, Kang DH, W Choi C, Kim SJ, et al. Cimetropium bromide does not improve polyp and adenoma detection during colonoscope withdrawal: A randomized, double-blind, placebo-controlled study. Medicine (Baltimore). 2018 Jun;97(25): e11253.

12 Barclay RL, Vicari JJ, Doughty AS, Johanson JF, Greenlaw RL. Colonoscopic withdrawal times and adenoma detection during screening colonoscopy. N Engl J Med. 2006 Dec; 355(24):2533-41.

13 Denzer U, Beilenhoff U, Eickhoff A, Faiss S, Huttl P, In der Smitten S, et al. Deutsche Gesellschaft fur Gastroenterologie V-uS [S2k guideline: quality requirements for gastrointestinal endoscopy, AWMF registry no. 021022]. Z Gastroenterol. 2015 Dec;53(12):1496530 .

14 Shaukat A, Church TR, Shanley R, Kauff ND, O'Brien MJ, Mills GM, et al. Development and validation of a clinical score for predicting risk of adenoma at screening colonoscopy. Cancer Epidemiol Biomarkers Prev. 2015 Jun; 24(6):913-20.

15 Lee CK, Park DI, Lee SH, Hwangbo Y, Eun CS, Han DS, et al. Participation by experienced endoscopy nurses increases the detection rate of colon polyps during a screening colonoscopy: a multicenter, prospective, randomized study. Gastrointest Endosc. 2011 Nov;74(5):1094-102.

16 Aslanian HR, Shieh FK, Chan FW, Ciarleglio MM, Deng Y, Rogart JN, et al. Nurse observation during colonoscopy increases polyp detection: a randomized prospective study. Am J Gastroenterol. 2013 Feb;108(2):166-72.

17 Sanaka MR, Deepinder F, Thota PN, Lopez R, Burke CA. Adenomas are detected more often in morning than in afternoon colonoscopy. Am J Gastroenterol. 2009 Jul;104(7):1659-64.

18 Kondo S, Yamaji Y, Watabe H, Yamada A, Sugimoto T, Ohta M, et al. A randomized controlled trial evaluating the usefulness of a transparent hood attached to the tip of the colonoscope. Am J Gastroenterol. 2007 Jan; 102(1):75-81.

19 Bronzwaer ME, Dekker E, Weingart V, Groth S, Pioche M, Rivory J, et al. Feasibility, safety, and diagnostic yield of the Extra Wide Angle View (EWAVE) colonoscope for the detection of colorectal lesions. Endoscopy. 2018 Jan;50(1):63-8.

$20 \mathrm{Ng}$ SC, Tsoi KK, Hirai HW, Lee YT, Wu JC, Sung JJ, et al. The efficacy of cap-assisted colonoscopy in polyp detection and cecal intubation: a meta-analysis of randomized controlled trials. Am J Gastroenterol. 2012 Aug; 107(8):1165-73.
21 Triantafyllou K, Polymeros D, Apostolopoulos P, Lopes Brandao C, Gkolfakis P, Repici A, et al. Endocuff-assisted colonoscopy is associated with a lower adenoma miss rate: a multicenter randomized tandem study. Endoscopy. 2017 Nov;49(11):1051-60.

22 Bevan R, Ngu WS, Saunders BP, Tsiamoulos Z, Bassett P, Hoare Z, et al. The ADENOMA Study. Accuracy of Detection using Endocuff Vision ${ }^{\mathrm{TM}}$ Optimization of Mucosal Abnormalities: study protocol for randomized controlled trial. Endosc Int Open. 2016 Feb; 4(2):E205-12.

23 Floer M, Biecker E, Fitzlaff R, Röming $H$, Ameis D, Heinecke A, et al. Higher adenoma detection rates with endocuff-assisted colonoscopy - a randomized controlled multicenter trial. PLoS One. 2014 Dec; 9(12):e114267.

24 Lenze F, Beyna T, Lenz P, Heinzow HS, Hengst K, Ullerich H. Endocuff-assisted colonoscopy: a new accessory to improve adenoma detection rate? Technical aspects and first clinical experiences. Endoscopy. 2014 Jul; 46(7):610-4.

25 Dik VK, Gralnek IM, Segol O, Suissa A, Belderbos TD, Moons LM, et al. Multicenter, randomized, tandem evaluation of EndoRings colonoscopy-results of the CLEVER study. Endoscopy. 2015 Dec;47(12):1151-8.

26 Gralnek IM, Suissa A, Domanov S. Safety and efficacy of a novel balloon colonoscope: a prospective cohort study. Endoscopy. 2014 Oct; 46(10):883-7.

27 Halpern Z, Gross SA, Gralnek IM, Shpak B, Pochapin M, Hoffman A, et al. Comparison of adenoma detection and miss rates between a novel balloon colonoscope and standard colonoscopy: a randomized tandem study. Endoscopy. 2015 Mar;47(3):238-44.

28 Hasan N, Gross SA, Gralnek IM, Pochapin M, Kiesslich R, Halpern Z. A novel balloon colonoscope detects significantly more simulated polyps than a standard colonoscope in a colon model. Gastrointest Endosc. 2014 Dec;80(6): 1135-40.

29 Subramanian V, Mannath J, Hawkey CJ, Ragunath K. High definition colonoscopy vs. standard video endoscopy for the detection of colonic polyps: a meta-analysis. Endoscopy. 2011 Jun;43(6):499-505.

30 Omata F, Ohde S, Deshpande GA, Kobayashi D, Masuda K, Fukui T. Image-enhanced, chromo, and cap-assisted colonoscopy for improving adenoma/neoplasia detection rate: a systematic review and meta-analysis. Scand J Gastroenterol. 2014 Feb;49(2):222-37. 
31 Leung WK, Lo OS, Liu KS, Tong T, But DY, Lam FY, et al. Detection of colorectal adenoma by narrow band imaging (HQ190) vs. high-definition white light colonoscopy: a randomized controlled trial. Am J Gastroenterol. 2014 Jun;109(6):855-63.

32 Gralnek IM. Emerging technological advancements in colonoscopy: Third Eye ${ }^{\circledR}$ Retroscope ${ }^{\circledR}$ and Third Eye ${ }^{\circledR}$ Panoramic(TM), Fuse ${ }^{\circledR}$ Full Spectrum Endoscopy ${ }^{\circledR}$ colonoscopy platform, Extra-Wide-Angle-View colonoscope, and NaviAid(TM) G-EYE(TM) balloon colonoscope. Dig Endosc. 2015 Jan; 27(2):223-31.

33 Papanikolaou IS, Apostolopoulos P, Tziatzios G, Vlachou E, Sioulas AD, Polymeros D, et al. Lower adenoma miss rate with FUSE vs. conventional colonoscopy with proximal retroflexion: a randomized back-to-back trial. Endoscopy. 2017 May;49(5):468-75

34 Alagappan M, Brown JR, Mori Y, Berzin TM. Artificial intelligence in gastrointestinal endoscopy: the future is almost here. World J Gastrointest Endosc. 2018 Oct;10(10):23949.

35 Fernández-Esparrach G, Bernal J, LópezCerón M, Córdova H, Sánchez-Montes C, Rodríguez de Miguel C, et al. Exploring the clinical potential of an automatic colonic polyp detection method based on the creation of energy maps. Endoscopy. 2016 Sep;48(9):837-42.
36 Wang Y, Tavanapong W, Wong J, Oh JH, de Groen PC. Polyp-Alert: near real-time feedback during colonoscopy. Comput Methods Programs Biomed. 2015 Jul;120(3):164-79.

37 Misawa M, Kudo SE, Mori Y, Nakamura H, Kataoka S, Maeda Y, et al. Characterization of Colorectal Lesions Using a Computer-Aided Diagnostic System for Narrow-Band Imaging Endocytoscopy. Gastroenterology. 2016 Jun; 150(7):1531-1532.e3.

38 Byrne MF, Chapados N, Soudan F, Oertel C, Linares Pérez M, Kelly R, et al. Real-time differentiation of adenomatous and hyperplastic diminutive colorectal polyps during analysis of unaltered videos of standard colonoscopy using a deep learning model. Gut. 2019 Jan; 68(1):94-100.

39 Klare P, Sander C, Prinzen M, Haller B, Nowack S, Abdelhafez M, Poszler A, Brown H, Wilhelm D, Schmid RM, von Delius S, Wittenberg T: Automated polyp detection in the colorectum: a prospective study (with videos). Gastrointest Endosc. 2019 Mar;89(3):576-582.e1.

40 Kudo S, Tamura S, Nakajima T, Yamano H, Kusaka H, Watanabe H. Diagnosis of colorectal tumorous lesions by magnifying endoscopy. Gastrointest Endosc. 1996 Jul;44(1):8-14.
41 Mori Y, Kudo SE, Wakamura K, Misawa M, Ogawa Y, Kutsukawa M, et al. Novel computer-aided diagnostic system for colorectal lesions by using endocytoscopy (with videos). Gastrointest Endosc. 2015 Mar;81(3):621-9.

42 Misawa M, Kudo SE, Mori Y, Cho T, Kataoka S, Yamauchi A, Ogawa Y, Maeda Y, Takeda K, Ichimasa K, Nakamura H, Yagawa Y, Toyoshima N, Ogata N, Kudo T, Hisayuki T, Hayashi T, Wakamura K, Baba T, Ishida F, Itoh $\mathrm{H}$, Roth $\mathrm{H}$, Oda M, Mori K: Artificial Intelligence-Assisted Polyp Detection for Colonoscopy: Initial Experience. Gastroenterology. 2018 Jun;154(8):2027-2029.e3.

43 Sosna J, Morrin MM, Kruskal JB, Lavin PT, Rosen MP, Raptopoulos V. CT colonography of colorectal polyps: a metaanalysis. AJR Am J Roentgenol. 2003 Dec;181(6):1593-8.

44 Leung F, Kulasingam V, Diamandis EP, Hoon DS, Kinzler K, Pantel K, et al. Circulating Tumor DNA as a Cancer Biomarker: fact or Fiction? Clin Chem. 2016 Aug;62(8):1054-60.

45 Heitzer E, Haque IS, Roberts CE, Speicher MR. Current and future perspectives of liquid biopsies in genomics-driven oncology. Nat Rev Genet. 2019 Feb;20(2):71-88.

46 Heitzer E, Perakis S, Geigl JB, Speicher MR. The potential of liquid biopsies for the early detection of cancer. NPJ Precis Oncol. 2017 Oct;1(1):36.

47 Perakis S, Speicher MR. Emerging concepts in liquid biopsies. BMC Med. 2017 Apr;15(1): 